J. Miner. Stoffwechs. Muskuloskelet. Erkrank. $2018 \cdot 25: 82-89$

https://doi.org/10.1007/s41970-018-0041-6

Online publiziert: 25. Juli 2018

(c) Der/die Autor(en) 2018

CrossMark
Nicole Gabriele Grün · Nicholas Donohue · Patrick Holweg · Annelie-

Martina Weinberg

Universitätsklinik für Orthopädie und Traumatologie, Medizinische Universität Graz, Graz, Österreich

\title{
Resorbierbare Implantate in der Unfallchirurgie
}

\begin{abstract}
Eine Vision in der Orthopädie und Traumatologie ist die Entwicklung von resorbierbaren Werkstoffen, die funktionelle Eigenschaften und gleichzeitig keine Nebenwirkungen oder Langzeitschäden im menschlichen Körper aufweisen. Heutzutage steigt die Zahl der Sport- und Freizeitverletzungen stetig an und betrifft dabei vorwiegend junge Patienten.
\end{abstract}

Vor allem in diesem Patientengut ist der Wunsch nach Vermeidung einer zweiten Operation und einem restitutio ad integrum dominierend. Im Gegensatz $\mathrm{zu}$ jungen Menschen weisen geriatrische Patienten andere Problematiken auf: $\mathrm{Ne}$ ben der schlechten Wundheilung ist die Frakturheilung nach der Durchführung einer Osteosynthese oftmals verzögert oder bleibt vollständig aus. Darüber hinaus ist der ältere Patient gefährdet, Infektionen primär oder auch sekundär zu erleiden. Letzteres tritt bei geschwächtem Immunsystem auf und ist auf den Biofilm unterhalb des Implantates zurückzuführen. Hinsichtlich der Genese gibt es verschiedene Ursachen. Im geriatrischen Patientengut wäre ein die Heilung unterstützender Werkstoff von Vorteil, der nicht nur antibakterielle, sondern sowohl osteokonduktive als auch osteoinduktive Eigenschaften aufweist. Fasst man diese Bedingungen zusammen, so sollte das ideale Implantat die Frakturheilung funktionell unterstützen (osteoinduktive Eigenschaft), Infektionen minimieren oder verhindern und sich vollständig ohne gesundheitsschädigende Rückstände auflösen, damit eine optimale Versorgung garantiert werden kann. Aus ökonomischer Sicht wäre die
Reduktion von Infektionen, die bessere Heilung und der Wegfall der Implantatentfernung insbesondere im Kindesund Jugendalter ein anzustrebendes Ziel.

Der Goldstandard in der Orthopädie und Unfallchirurgie ist der Einsatz von nichtresorbierbaren Implantaten, die aus Titan, Kobalt-Chrom, Zirkonium oder Stahl hergestellt und für die Osteosynthese bei Frakturen verwendet werden. Diese Materialien sind allerdings zu rigide, aber lasttragend, und weisen eine gute Biokompatibilität auf. Letzteres wird jedoch zunehmend in Frage gestellt. Obwohl diese Materialien nicht korrodieren, geben sie Präzipitationen ab. Bereits 2002 zeigte eine Gruppe aus Amerika, dass Titanpartikel einen Einfluss auf die Differenzierung von Osteoblasten haben. Dabei wurden mesenchymale Stammzellen aus Hüftköpfen isoliert und zeigten nicht nur eine verminderte Proliferation und Lebensfähigkeit, sondern auch eine reduzierte Expression von Knochenformationsfaktoren in Anwesenheit von Titanpartikeln. Die Wissenschaftler schlussfolgerten daraus, dass eine Langzeitexposition mit Titan in vivo die Knochenformation im Interface von Knochen und Implantat reduziert, was die Biofilmbildung erhöht [1]. Zusätzlich weist die FDA darauf hin, dass es einen Zusammenhang zwischen Titanimplantaten und einer möglichen Metallsensitivität oder dem Ausbruch allergischer Reaktionen geben kann [2, 3]

Ein weiterer Nachteil, das sog. „stress shielding ", tritt bei der Osteosynthese mit herkömmlichen Implantaten aufgrund unterschiedlicher Elastizitätsmodule von Knochen und Implantat auf (• Abb. 1; [4]). Bei der Osteosynthese mit Platten baut sich der Knochen unter der Platte ab, weil an dieser Stelle die Kraft über das Implantat getragen wird. Somit muss der Knochen keine oder nur wenig Arbeit selbst verrichten, wodurch die Remodellingaktivität im Sinne eines Abbaus des Knochens unterhalb des Implantates verstärkt wird. Daraus resultieren vermehrt Frakturen am Übergang zwischen Platte und Knochen (periprothetische/ implantatassoziierte Fraktur) oder nach Entfernung der Osteosynthese. Letzteres entspricht einer Refraktur, d.h. eine Fraktur an der gleichen Stelle [5, 6].

Speziell in der Kinder- und Jugendchirurgie haben konventionelle Werkstoffe den Nachteil, dass der Knochen über die Implantate wächst, diese nach einigen Jahren nicht mehr konventionell entfernt werden können und der Knochen ggf. künstlich gebrochen oder aufgebohrt werden muss.

Daher sind an sich verträgliche Materialien im herkömmlichen Sinn durchaus kritisch zu hinterfragen und entsprechen bei weitem nicht den Anforderungen an ein optimales Implantat.

\section{Biomaterialien: resorbierbar und biokompatibel}

Allgemein spricht man von Biomaterialien, wenn diese die Arbeit der Organe unterstützen oder ersetzen. Zurzeit werden Implantate oder Füllungen aus Biomaterialien vorwiegend im Bereich der Zahn-, Herz-, Gehirn- oder Knochenchirurgie appliziert [7-9]. Diese sind nicht notwendigerweise resorbierbar.

In der Orthopädie und Unfallchirurgie sollte das optimale Implantat für die Versorgung von Brüchen resorbierbar sein. Neben der nötigen Streckfestigkeit (• Abb. 1; ähnlich dem Knochen) 


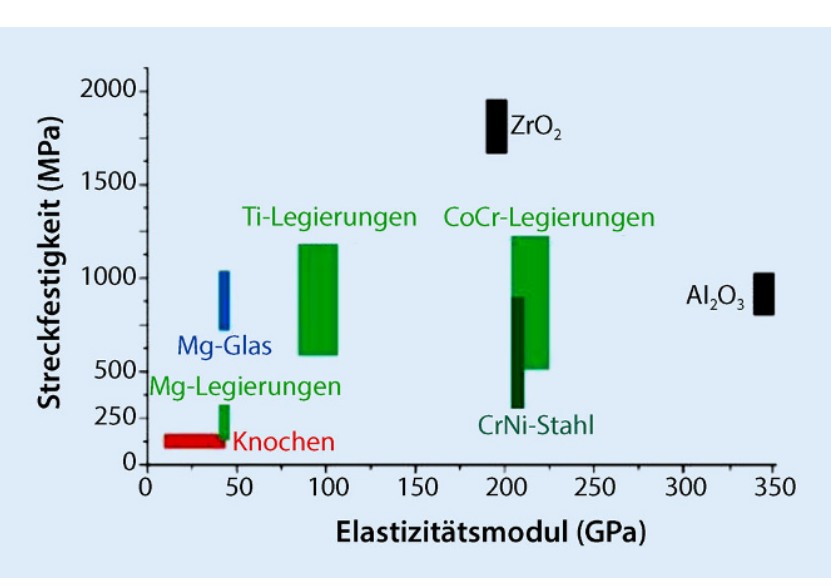

Abb. $1<$ Das ideale resorbierbare Implantat. Die Streckfestigkeit sollte ähnlich dem Knochen sein zur Frakturstabilisierung sollte das Implantat homogen degradieren, sodass keine Implantatrückstände mehr nachweisbar sind. Wie bereits erwähnt sollten Implantate aus bioresorbierbaren Werkstoffen eine gute Biokompatibilität aufweisen und zusätzlich die Heilung funktionell unterstützen. Hierbei sollten jene Elemente, die bei der Degradation des Implantats frei werden, die Heilung fördern, den Knochen unterhalb des Implantates nicht abbauen und damit die Gefahr von erneuten Frakturen verhindern. Im Folgenden können die einzelnen Implantatwerkstoffe nur beispielhaft aufgezeigt und ihre Vor- und Nachteile nur beispielhaft gegenüber gestellt werden.

\section{Polymere}

Es gibt verschiedene resorbierbare Werkstoffe, die als Implantate oder Gewebeersatz zum Einsatz kommen. In der Literatur werden vor allem polymerbasierte Implantate aus zuckerhaltigen Derivaten (Poly[L-lactid] [PLLA], Poly[lactid-co-glycolid] [PLGA], Poly[DLlactid] [PDLLA]) genannt (• Abb. 2). Die Forschung konnte diese Werkstoffe in den letzten Jahren hinsichtlich ihrer Abbaugeschwindigkeit, aber auch bezüglich ihrer mechanischen Eigenschaften optimieren, jedoch wurden die damit verbundenen Probleme nach wie vor nicht zur Gänze gelöst:

a. Polymerbasierte Werkstoffe benöti-

gen für den Abbau Enzyme, welche jedoch in vivo nur begrenzt zur Verfügung stehen. Daraus resultiert eine Degradationszeit der Polymere zwischen 2 und 10 Jahren.

b. Polymerbasierte Systeme sind brüchig und haben ein niedriges Elastizitätsmodul (E-Modul). Der Einsatz von Polymeren beschränkt sich daher auf nicht lasttragende Regionen und kann daher nicht für alle Frakturversorgungen eingesetzt werden.

c. Polymerbasierte Werkstoffe können aufgrund der langen Resorptionsdauer chronische, aseptische Entzündungen hervorrufen, da diese Implantate dann über die Zeit ähnlich wie ein Fremdkörper im menschlichen Gewebe wirken: Die humorale und zelluläre Immunantwort führen zur Bildung einer fibrotischen Kapsel. Dadurch verzögert sich die Resorption zusätzlich und in manchen Fällen kommt es zum Wandern des Implantates an die Oberfläche.

Eine Alternative zu den polymerbasierten Zuckermolekülen ist zum Beispiel die Polyhydroxybuttersäure (PHB). Die Herstellungsprozesse erfolgen über Bakterien, wodurch die Toxizität hierbei zu einem Problem werden kann. Jedoch konnte man die Toxizität des Materials in den letzten Jahren deutlich reduzieren, was den Einsatz von PHB-Implantaten in der Medizin ermöglichte [10]. Das Problem der Metabolisierung aufgrund begrenzter Enzyme bleibt jedoch - wie bei den polymerbasierten Werkstoffen - erhalten. Wissenschaftler beschäftigen sich daher mit der Zulegierung von Enzymen, um eine adäquate Resorption des polymeroder PHB-basierten Implantates gewährleisten zu können. Diese Forschungen stehen aber noch am Anfang und somit ist ihr Einsatz noch weit von einer klinischen Zulassung in der Unfallchirurgie entfernt. Zusätzlich muss festgehalten werden, dass bis heute kein funktioneller Effekt (osteoinduktiv oder -konduktiv) auf das Remodelling des Knochens durch die freigesetzten Stoffe von biodegradierbaren Polymer- oder PHB-basierten Implantaten nachgewiesen werden konnte.

\section{Komposite}

Als Komposite bezeichnet man die $\mathrm{Zu}-$ sammensetzung aus heterogenen Einzelbestandteilen, die entweder rein organischer, rein anorganischer oder gemischter Herkunft sein können. Diese bioaktiven Gläser sind biokompatibel, osteokonduktiv und konvertieren in vivo $\mathrm{zu}$ Hydroxylapatit (HA) [11]. Um eine biologische Wirksamkeit zu erreichen, werden diese Materialien meist mit Zellen oder Wirkstoffen gekoppelt, um die Osteokonduktivität, aber vor allem die Osteoinduktivität einzuleiten. Diese bioaktiven Gläser sind in der Lage, an hartes und weiches Gewebe zu binden und sich mit der Zeit aufzulösen. Der Nachteil besteht jedoch in den geringen biomechanischen Eigenschaften. Sie sind zumeist spröde und zeigen eine geringe Festigkeit, wobei diese dem menschlichen trabekulären Knochen oftmals ähnelt [12]. Um die biomechanischen Eigenschaften zu verbessern, werden Beschichtungen aus Polycaprolactonen oder Polylactiden verwendet $[13,14]$. Dadurch wird die Lasttragung auf die sehr stabilen Polymere übertragen, was aber wiederum eine geringe Porosität voraussetzt. Diese geringere Porosität kann jedoch dazu führen, dass Knochenzellen schlechter infiltrieren können und somit zelluläre und molekulare Vorgänge in geringerem Ausmaß stattfinden, was zusätzlich die Osteointegration stört [11].

Das bekannteste Komposit, welches am Markt erhältlich ist, besteht aus HA und Siliziumoxid, welches in einem Sol-Gel-Verfahren hochporös hergestellt wird. Obwohl die Porosität sehr hoch ist, hat dieses spezielle Komposit sehr gute biomechanische Eigenschaften und eine Belastbarkeitsgrenze von $40 \mathrm{MPa}$ [15]. Jedoch kann es als Implantatma- 
terial in lasttragenden Regionen nicht eingesetzt werden, weil die hohe mechanische Stabilität des kortikalen Knochens (100-150 MPa) den des Materials weit übersteigt. Diese Materialien werden derzeit bei der Auffüllung von Defekten klinisch verwendet.

\section{Biokeramik}

Keramik zählt zusammen mit den Polymeren und Kompositen zu den synthetischen Knochenersatzmaterialien. Neben ihrer Biokompatibilität weisen diese Materialien auch eine gute Osteokonduktivität, Osteointegration und nichtimmunogene Wirkung auf. Biokeramiken setzen sich aus synthetischen Stoffen wie HA, Alpha- und Beta-Tricalciumphosphaten (TCP) zusammen. Dabei können diese Implantate einerseits aus reinem HA bestehen oder auch biphasische Produkte aus HA und Beta-TCP sein. Reine HAKeramiken sind im Vergleich zum natürlich vorkommenden HA weitgehend chemisch identisch, erzeugen die Freisetzung von Calcium und Phosphat und eine Verbundsosteogenese. Um die Osteointegration zu verbessern, können reine HAKeramiken beschichtet werden. Jedoch muss an dieser Stelle erwähnt werden, dass reines HA und somit reine HAKeramiken für einige Jahre im Körper verweilen, bevor sie vollständig abgebaut sind [16]. Als Implantate für die Versorgung von Brüchen erscheint ihr Einsatz bisher ebenfalls nicht geeignet.

Biphasische Produkte aus HA und Beta-TCP besitzen einen langsamen und schnellen Degradationsanteil, wodurch das Knochenwachstum erleichtert und die Stabilität erhalten bleibt. Der Nachteil der TCPs sind jedoch die schlechten biomechanischen Eigenschaften. Die Calciumphosphate zeigen sich hierbei als spröde und wenig zugfest (6-10 MPa), wodurch diese Materialien dazu neigen, auseinanderzubrechen [17]. Daher bleibt auch hier der Einsatz dieses Implantatmaterials in der Unfallchirurgie eingeschränkt.

Nichtsdestotrotz sind Biokeramiken aufgrund ihrer Biokompatibilität als medizinische Produkte geeignet und werden daher in nichtlasttragenden Bereichen wie im Mittelohr oder der Mund-

J. Miner. Stoffwechs. Muskuloskelet. Erkrank. 2018 · 25:82-89

https://doi.org/10.1007/s41970-018-0041-6

๑) Der/die Autor(en) 2018

\section{N. G. Grün · N. Donohue · P. Holweg · A.-M. Weinberg}

\section{Resorbierbare Implantate in der Unfallchirurgie}

\section{Zusammenfassung}

Resorbierbare Implantate sind eines der großen Ziele in der operativen, orthopädischen und traumatologischen Chirurgie. Die Vorteile dieser resorbierbaren Werkstoffe liegen auf der Hand: Belastende, mögliche Implantatentfernungen fallen weg und somit auch die damit verbundenen Komplikationen. Mittlerweile weist eine wachsende Anzahl von Patienten bei konventionellen Titanimplantaten eine Metallsensitivität auf, die in ihren Folgen noch nicht gänzlich abschätzbar ist. Hinzu kommt das ethische Bedürfnis eines jeden Menschen, unversehrt bis ins hohe Alter zu bleiben und ein restitutio ad integrum nach Verletzungen zu erreichen.

Dies führt dazu, dass resorbierbare Implantate wie PLLA (Poly[L-lactid]) und PLGA (Poly[lactid-co-glycolid]) immer mehr Anwendung finden, jedoch aufgrund ihrer Brüchigkeit für die lasttragende Stabilisierung von Brüchen nicht geeignet sind. Ähnlich sieht die Situation bei Kompositen und Biokeramiken aus. All diese synthetischen Knochenersatzmaterialien sind sehr gut biokompatibel, aber nur für den Einsatz in nichtlasttragenden Regionen geeignet.
Magnesiumimplantate sind seit Langem eine Alternative, hatten aber bisher den Nachteil, dass die Degradation nicht ausreichend gesteuert werden konnte und die damit eintretende Pitkorrosion zu einer Gasbildung beim Abbau führt. Trotz der Gasbildung zeigen Studien eine vollständige, sehr hohe Heilungsrate auf, welche auf die mittlerweile nachgewiesene, sehr gute Osteoinduktion von Magnesium zurückzuführen ist. Aufgrund dieser Eigenschaften kann Magnesium auch zur Herstellung von Implantaten für den pathologischen Knochen eingesetzt werden. Welche resorbierbaren Werkstoffe derzeit in der Klinik eingesetzt werden und welche Vor- bzw. Nachteile diese bringen wird in dem folgenden Artikel detailliert dargestellt. Forschungsergebnisse mit unterschiedlichen Magnesiumlegierungen und neue Anwendungsmöglichkeiten von Magnesiumimplantaten im pathologischen Knochen werden diskutiert.

Schlüsselwörter

Resorbierbar · Biokompatibel · Knochen .

Magnesium · Osteoporose

\section{Resorbable implants in trauma surgery}

\section{Abstract}

Bioresorbable implants are currently one of the main goals in orthopedic and trauma surgery. The advantages are apparent: potentially difficult implant removal operations are unnecessary, thereby avoiding complications. A growing number of patients exhibit metal sensitivity to implants, the long-term consequences of which remain to be determined. Additionally, there is an ethical requirement, that every person should reach old age undamaged and be completely restored following injury.

As a result, resorbable implants such as PLLA/PLGA are being used more frequently, although their brittleness makes them unsuitable for load-bearing fracture stabilization. Composites and bioceramics face similar problems. All of these synthetic bone substitutes are highly biocompatible, but only suitable for use in nonload-bearing regions. Magnesium implants have been an alternative for a long time, although until now, they had the disadvantage that degradation could not be controlled sufficiently and the resulting pit corrosion lead to gas formation during degradation. Despite this gas formation, studies have shown a very high, complete healing rate, which is caused by the excellent, proven osteoinduction of magnesium. Due to these properties, magnesium can also be used for the production of implants for pathological bone conditions.

\section{Keywords}

Resorbable - Biocompatible Bone . Magnesium · Osteoporosis 
höhle eingesetzt oder als Beschichtung für konventionelle Materialien verwendet, um eine bessere Osteoinduktion im Interface, zum Beispiel in Prothesen, zu gewährleisten [18].

\section{Biodegradierbare Metalle}

Biodegradierbare Metalle wie Eisen (Fe), Zink $(\mathrm{Zn})$ und Magnesium $(\mathrm{Mg})$ sind im Vergleich zu anderen Biomaterialien sehr zugfest, stabil und lasttragend $[19,20]$. Als Basiswerkstoff ist Eisen von großem Interesse, weil es einen niedrigen Schmelzpunkt besitzt und somit relativ einfach durch Schmelzen oder Spritzguss unter Luftatmosphäre und Wärmeumformung verarbeitet werden kann. Untersuchungen, die von unserer Forschungsgruppe getätigt wurden [21], konnten allerdings zeigen, dass der notwendige Sauerstoff, der für die Korrosion und den damit verbundenen Abbau von eisenhaltigen Implantaten benötigt wird, nicht in einem ausreichenden Maß vor Ort vorhanden ist. Daraus resultieren lange Abbauzeiten, wodurch eisenbasierte Implantate nur eingeschränkt resorbierbar sind und Eisen somit derzeit kein geeignetes resorbierbares Material darstellt [22]. Im Gegensatz zu Eisen hat Zink den Nachteil, dass es eine zu niedrige Härte und Verformbarkeit aufweist. Zudem ist Zink ein Korrosionsverhinderer, wodurch dieses Metall gerne für die $\mathrm{Zu}$ legierung von zum Beispiel magnesiumbasierten Implantaten verwendet wird.

Magnesium weist neben der hervorragenden Biokompatibilität auch gute biomechanische Eigenschaften auf. Vorversuche im Tier haben sehr gute mechanische Ergebnisse der magnesiumbasierten Implantate im Vergleich zu anderen Werkstoffen beim Push-out-Test gezeigt. Weitere wissenschaftliche Arbeiten demonstrierten, dass Magnesium osteoinduktiv ist und damit zum ersten Mal auch die Heilung unterstützt [23-25]. Im letzten Jahrzehnt wurden magnesiumbasierte Implantate ausreichend untersucht, je- doch wurden meist Legierungen genutzt, die z. B. seltene Erden, Yttrium oder Gadolinium [26-29] enthielten. Diese Zulegierungen haben den Vorteil, die Korrosionsgeschwindigkeit zu verlangsamen und dadurch das Implantat stabiler $\mathrm{zu}$ machen.

In den Anfangsstudien unserer Forschungsgruppe enthielten die magnesiumbasierten Implantate noch zulegierte seltene Erden (WE43; Mg-Y-Nd-HRE). In diesen Studien zeigten sich ein starkes Knochen-Implantat-Interface und eine gute Osteointegration im wachsenden Rattenmodell. Dabei wurde WE43 mit Titan als konventionelles Implantatmaterial verglichen und daraus gefolgert, dass der Knochen auf das magnesiumbasierte Implantat mit Knochenanbau reagierte und somit eine wichtige Eigenschaften für den Einsatz als Implantat zur Fixierung von Knochenbrüchen aufweist [30]. In einer weiteren Studie wurde das magnesiumbasierte Implantat WZ21 $(\mathrm{Mg}$, $1 \% \mathrm{Zn}, 0,25 \% \mathrm{Ca}, 0,15 \% \mathrm{Mn}, 2 \% \mathrm{Y}$ )

Hier steht eine Anzeige.

\section{基 Springer}




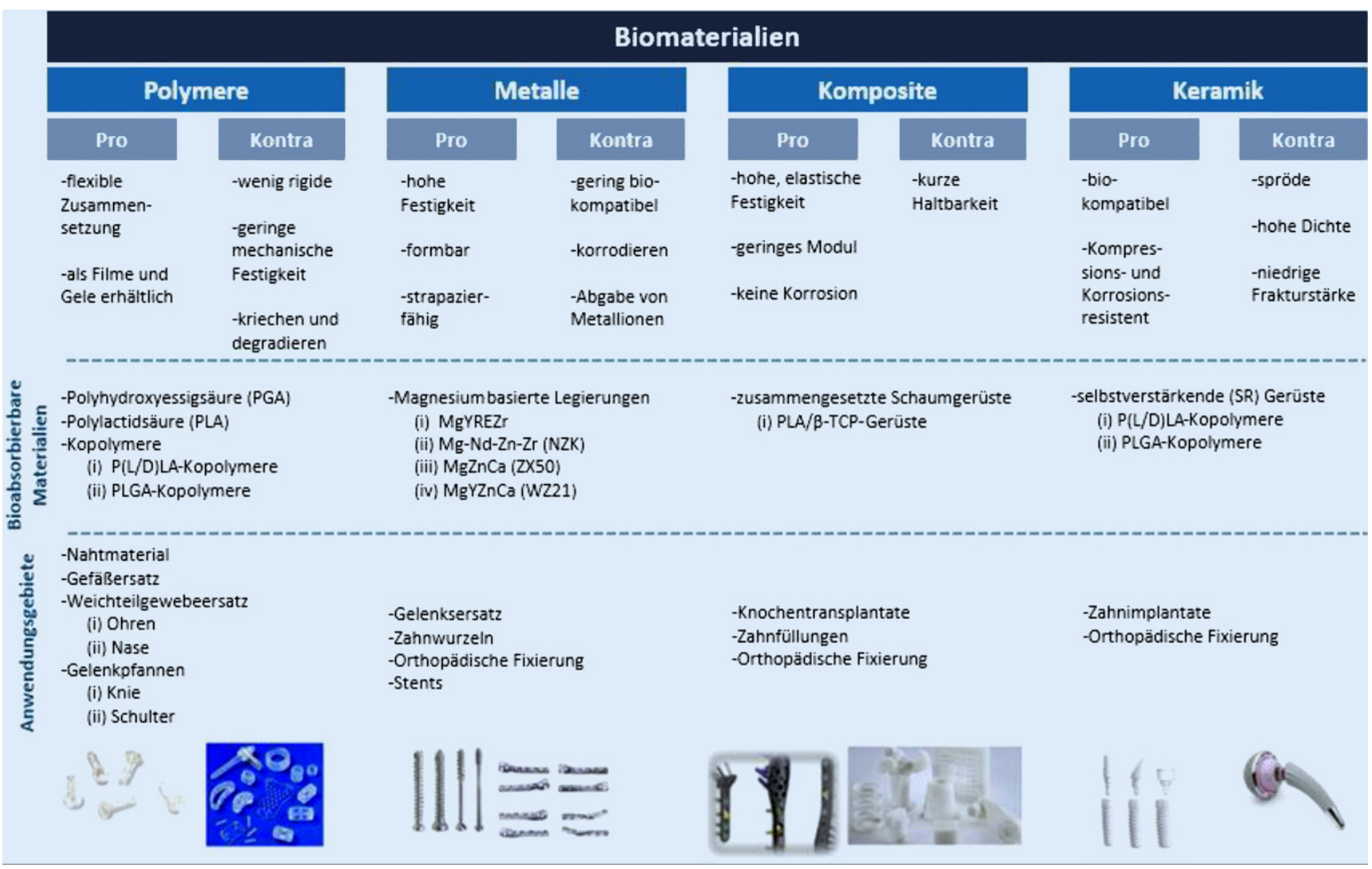

Abb. 2 A Biomaterialien in der Medizin. Vor- und Nachteile der verschiedenen Werkstoffe inklusive detaillierte Aufstellung der einzelnen Materialien gruppiert in Polymere, Metalle, Komposite und Keramik. Anwendungsgebiete der Werkstoffe zeigen die Vielfältigkeit und den Bedarf an neuen bioresorbierbaren Materialien

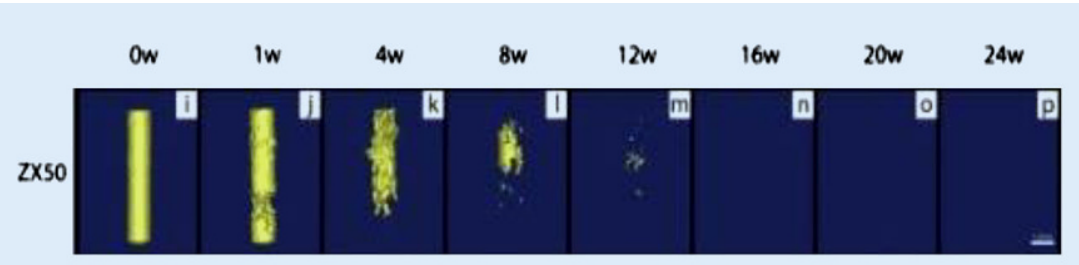

Abb. 3 A Degradationsverhalten von ZX50 über einen Zeitraum von 24 Wochen. Nach den In-vivo- $\mu \mathrm{CT}$-Scans wurde eine 3D-Rekonstruktion der Pins angefertigt. (Adaptiert von Kraus [31])

verwendet, um den Degradationsverlauf des Implantatmaterials mittels $\mu \mathrm{CT}$ über einen Zeitraum von 24 Wochen aufzuzeichnen. Hierbei wurden keine Entzündungsreaktionen direkt nach der Implantation und im weiteren Verlauf beobachtet. Die langsame Degradation von WZ21 hatte keine Auswirkung auf den Knochenanbau. Speziell zwischen 4 und 8 Wochen nach Implantation wurde eine vermehrte Knochenbildung beobachtet [31].

Bei der Forschung und Herstellung von resorbierbaren Implantaten im Kindesalter muss man berücksichtigen, dass die eingesetzten Materialien keine Langzeitschäden hervorrufen sollten, da die $\mathrm{zu}$ erwartende Lebenszeit beträchtlich ist. Eigene Arbeiten [29] konnten zeigen, dass die intermetallischen Phasen bei zulegierten Elementen über Jahre noch nachweisbar waren und folglich Präzipitationen abgeben, wobei deren schädigende Wirkung nicht beurteilt werden kann. Ziel war es, ein resorbierbares Metall ohne seltene Erden zu legieren. Dies wurde in den letzten Jahren an der Medizinischen Universität in Graz in Zusammenarbeit mit der ETZ Zürich sowohl in vitro als auch in vivo in verschiedenen Tiermodellen getestet. Neben der sehr guten Verträglichkeit konnten auch die biomechanischen Eigenschaften durch verschiedene Prozesse in der Herstellung erhalten bleiben und Nachteile wie ein schneller Abbau des Implantats reguliert werden. So wurden verschiedene Zusammensetzungen von magnesium-, calcium- und zinkbasierten Legierungen getestet. Anfangs wurde die Magnesiumlegierung ZX50 (Mg, 5\% $\mathrm{Zn}, 0,25 \% \mathrm{Ca}, 0,15 \% \mathrm{Mn}$ ) verwendet. In den In-vivo-Studien im Kleintiermodell konnte man anhand der $\mu \mathrm{CT}$-Scans feststellen, dass das ZX50-Material zu schnell degradierte (• Abb. 3) und somit für den Einsatz als orthopädisches Implantat mit dieser Abbaugeschwindigkeit nicht geeignet war [29, 31, 32].

Bei der gewünschten Abbaugeschwindigkeit entsteht eine adäquate Konzentration von Magnesiumhydroxid $\left(\mathrm{Mg}[\mathrm{OH}]_{2}\right)$ und Hydrogengas aufgrund der elektrochemischen Reaktion, die als Pitkorrosion bekannt ist. $\operatorname{Das} \mathrm{Mg}(\mathrm{OH})_{2}$ ist unlöslich und bildet eine Schutz- 

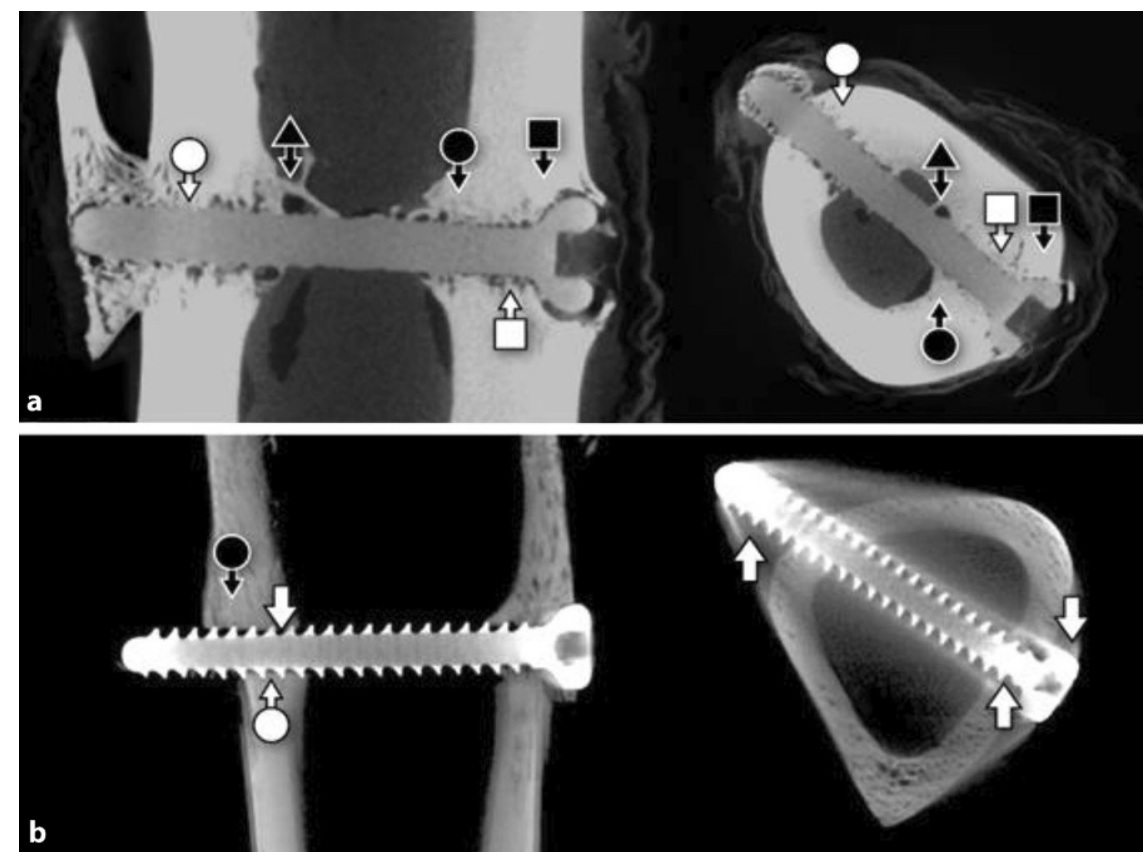

Abb. 4 ム Knochenanbau und Implantatdegradation in den frühen Phasen der Frakturheilung im Schaf. a Links: koronale Ansicht, rechts: axiale Ansicht auf die Tibia mit bikortikal implantierter ZX00-Schraube 12 Wochen nach Implantation. Endostale Knochenmasse und periostaler Knochenanbau inklusive neuer Knochenstrukturen innerhalb des Markraums (schwarze Pfeile); starkes Knochen-Implantat-Interface am Schraubenkopf am Schraubenende (weißePfeile). b Links: koronale Ansicht, rechts: axiale Ansicht auf die Tibia mit bikortikal implantierter Titanschraube 12 Wochen nach Implantation. Kallusformation (schwarzerPfeil); erwartetes, festes Interface (weißePfeile); metallische Artefakte von Titanschraube (weiße Pfeile ohne Kreis)

schicht um die Implantatoberfläche. Hebt sich der Chloridspiegel im Körper, so bindet das Magnesium aus $\mathrm{Mg}(\mathrm{OH})_{2}$ an Chlorid, um $\mathrm{MgCl}_{2}$ zu bilden. Dieses Produkt ist löslich und kann somit sowohl transportiert als auch abgebaut werden [33]. Dies bedeutet aber auch, dass eine ausgewogene Degradation vorhanden sein muss, da ansonsten das entstehende Gas zu einer Verdrängung des Knochens beiträgt und somit negative Auswirkungen hat. Dies führt auch dazu, dass ein zu schneller Abbau das Implantat noch vor der vollständigen Ausheilung der Knochenfraktur instabil werden ließe. Auf der anderen Seite kann eine zu langsame Degradation jedoch in einer chronischen, entzündlichen Immunreaktion, wie dies bei den polymerbasierten Materialien zu sehen ist, enden. Daher ist die Einstellung der Degradationsgeschwindigkeit eine Herausforderung für alle Wissenschaftler auf dem Gebiet der Implantatherstellung aus Magnesiumlegierungen und wird den Weg der magnesiumbasierten Implantatwerkstoffe und ihre Zukunft
ZX00 (Mg, 0,45\% Zn, 0,45\% Ca) wurde in die Tibia von Schafen implantiert und mit herkömmlichen Titanschrauben verglichen (•Abb.4).

Zwölf Wochen nach Implantation von ZX00 in die proximale Tibia der Schafe konnte gezeigt werden, dass neuer Knochen gebildet wird und sich ein stabiles Knochen-Implantat-Interface bildet (- Abb. 4a). Konventionelle Implantate wie Titan zeigen eine schwächere Knochenbildung unterhalb des Implantates (da die Last über die Schraube getragen wird; Knochen baut sich ab), was die Gefahr der Refraktur nach Metallentfernung in sich birgt. Oberhalb des Implantates zeigte sich ein guter Anbau, wobei die Osteokonduktivität von Magnesium in dieser Abbildung den Unterschied zu Titan mit bloßem Auge erkennen lässt.

Zusätzlich konnte in Rattenstudien gezeigt werden, dass sich das abgegebene Magnesium durch Diffusion und/oder Remodelling im kortikalen Knochen anlagert, wodurch der Knochen nahe der Frakturseite fester und härter wurde (- Abb. 5). Die dargestellten Ergebnisse zeigen einen guten Einsatz im Kindesalter auf, welcher aber durch klinische Studien erst bewiesen werden muss.

Der Einsatz im pathologischen (osteoporotischen) Knochen stellt eine Aufgabe für die Wissenschaft in der Zukunft dar. Gerade in diesem Bereich wäre ein funktionalisiertes Implantat von besonderer Bedeutung. Magnesium weist Eigenschaften auf, die gerade im Bereich der Infektion von Vorteil wären, und zusätzlich wäre die Sekundärinfektion reduzierbar, da sich das Implantat auflöst und somit nicht mehr ein Nährboden für Bakterien bei reduziertem Immungeschehen wäre.

Die World Health Organization (WHO) publizierte 3,5 Mio. spontane Fragilitätsfrakturen bei osteoporotischen Patienten in der Europäischen Union mit einer geschätzten wirtschaftlichen Belastung für vorausgehende und inzidente Fragilitätsfrakturen von $37 \mathrm{Mrd}$ € für 2010. Bis 2025 sollen die osteoporoseassoziierten Kosten um weitere $25 \%$ steigen. Zusätzlich prognostiziert die WHO eine Verdoppelung der über 60Jährigen und eine Verdreifachung der 


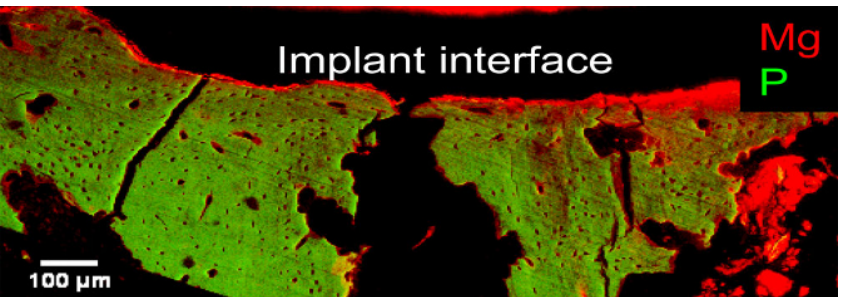

Abb. 5 A Verteilung von Magnesium und Phosphor, abgegeben von einem resorbierbaren Magnesiumimplantat. Magnesium (rot); Phosphor (grün). (Aus Grünewald et al. [36])

über 80-Jährigen mit dem Jahr 2050 [37].

In der Medizin sind verschiedene Subtypen der Osteoporose bekannt, die sich weitgehend in eine primäre und eine sekundäre Form zusammenfassen lassen. Unter primärer Osteoporose findet man den Typ 1, die sog. postmenopausale Osteoporose, und den Typ 2, die senile Osteoporose. $\mathrm{Zu}$ den chronischen Erkrankungen, die mit einer sekundären Osteoporose einhergehen, zählt man zum Beispiel das CushingSyndrom. Trotz dieser Unterteilungen ist die Charakterisierung der Osteoporose gleichbleibend. Im Laufe der Erkrankung kommt es zu einer Schwächung der trabekulären und kortikalen Knochenstruktur, wodurch sich der Knochen abbaut und $\mathrm{zu}$ einer reduzierten Mineralisierung und Zugfestigkeit führt; das Frakturrisiko steigt [38]. Diese pathologische Schwächung des Knochens beeinflusst dabei auch die biologischen und mechanischen Faktoren, die bei der Knochenheilung eine wesentliche Rolle spielen. Im gesunden Knochen sind die zellulären und molekularen Ereignisse bis ins kleinste Detail gut abgestimmt. Mesenchymale Vorläuferzellen differenzieren zu Osteoblasten, welche die Aufgabe besitzen, Knochen aufzubauen, vor allem an jener Stelle, die zuvor von Osteoklasten (differenzieren aus Monozyten-Makrophagen-Vorläufern) resorbiert wurde. Dieser Remodellingprozess startet mit dem Erwachsenenalter und ist im Laufe unseres Lebens ein kontinuierlicher Prozess. Man könnte es auch als ein orchestriertes Zusammenspiel von zellulären und molekularen Prozessen beschreiben. Im Gegensatz dazu findet man in der Osteoporose eine deutliche Abnahme an osteogenen Zellen und/oder die Abnahme der Aktivität dieser Zellen, während die Osteoklastenaktivität deutlich erhöht ist. Auf molekularer Ebene werden spontan entzündliche Zytokine, wie zum Beispiel Tumornekrosefaktor alpha (TNFa) oder Interleukine (IL-1 und -6), ausgeschüttet und Knochenformationsfaktoren wie Transformierender Wachstumsfaktor beta (TGF $\beta$ ) oder Insulinähnliche Wachstumsfaktoren (IGF1) herunterreguliert [6].

Die Heilung des Knochens nach der Implantation ist ein kritischer Punkt, wobei hier im Optimalfall der neu gebildete Knochen das Implantat umschließt und somit ein stabiles Knochen-ImplantatInterface entsteht [6]. Im pathologischen Knochen wie bei der Osteoporose wurde jedoch in In-vitro- und In-vivo-Experimenten gezeigt, dass die Osteointegration wesentlich später stattfindet [39-41]. Dem gegenüber stehen die magnesiumbasierten Implantate, die einen positiven Effekt auf die Knochenheilung haben, ein E-Modul ähnlich dem Knochen besitzen und im Gegensatz zu den polymerbasierten Werkstoffen eine sehr gute Verträglichkeit aufweisen [31, 42, 43]. Für diese osteoporotische Patientengruppe wurden bisher nur in sehr geringem Ausmaß Biomaterialien angeboten, da insbesondere die Belastbarkeit der Materialien nicht gegeben war und auch die Osteoinduktivität nur mäßig erreicht wurde. Da der derzeitige Stand der Wissenschaft nur auf die Vorteile von magnesiumbasierten Legierungen hinweisen kann und das biologische Wissen über den osteoporotischen Knochen noch nicht ausreichend ist, kann die Anwendung und Wirksamkeit von magnesiumbasierten Implantaten noch nicht gezeigt werden und bedarf intensiver Forschungsarbeit in den kommenden Jahren.

Zusammengefasst kann man feststellen, dass eine neue Dimension der
Knochenbruchheilung mit magnesiumbasierten Implantaten möglich wäre. Es ist das erste funktionelle Implantat, welches durch die kallusinduzierende Wirkung die Knochenbruchheilung aktiv unterstützt. Gleichzeitig weist es eine überlegene Biokompatibilität $\mathrm{zu}$ anderen Implantaten auf. Dieses in der Forschung nachgewiesene Phänomen muss sich nun in klinischen Studien beweisen. Es sind entsprechende Adaptionen der Legierungsoptimierung $\mathrm{zu}$ erwarten

\section{Korrespondenzadresse}

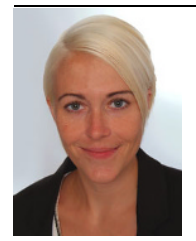

N. G. Grün, PhD

Universitätsklinik für Orthopädie und Traumatologie, Medizinische Universität Graz Auenbruggerplatz 5/6, $8036 \mathrm{Graz}$, Österreich nicole.gruen@medunigraz.at

Danksagung. Die Studien wurden im Rahmen des Laura - Bassi Centers of Excellence BRIC (Bioresorbable Implants in Children) durchgeführt und wurden von der FFG (Österreichische Forschungsförderungsgesellschaft) gefördert. Wir bedanken uns auch bei unserem Kooperationspartner Prof. Löffler (ETH Zürich) für die Bereitstellung der Materialien und deren stetige Weiterentwicklung. Ebenso bei Frau Prof. Lichtenegger (BOKU Wien) für die Testung der Knochenstrukturen.

Funding. Open access funding provided by Medica University of Graz.

\section{Einhaltung ethischer Richtlinien}

Interessenkonflikt. A.-M. Weinberg ist Stakeholder und CEO der Firma BRI.TECH GmbH. N. G. Grün, N. Donohue und P. Holweg geben an, dass kein Interessenkonflikt besteht.

Dieser Beitrag beinhaltet keine von den Autoren durchgeführten Studien an Menschen oder Tieren. Für die im Beitrag zitierten Studien gelten die jeweils dort angegebenen ethischen Richtlinien. Im Fall der nichtpublizierten Studien wird darauf verwiesen, dass der Großtierversuch (-Abb. 4) vom Österreichischen Bundesministerium für Wissenschaft, Forschung und Wirtschaft (GZ-Nummer: BMWFW-66.010/0073WF/V/3b/2015) bewilligt wurde.

Open Access Dieser Artikel wird unter der Creative Commons Namensnennung 4.0 International Lizenz (http://creativecommons.org/licenses/by/4.0/deed de) veröffentlicht, welche die Nutzung, Vervielfältigung, Bearbeitung, Verbreitung und Wiedergabe in jeglichem Medium und Format erlaubt, sofern Sie den/die ursprünglichen Autor(en) und die Quelle ordnungsgemäßnennen, einen Link zur Creative Commons Lizenz beifügen und angeben, ob Änderungen vorgenommen wurden. 


\section{Literatur}

1. Wang ML, Nesti LJ, Tuli R, Lazatin J, Danielson KG, Sharkey PF et al (2002) Titanium particles suppress expression of osteoblastic phenotype in human mesenchymal stem cells. J Orthop Res 20(6):1175-1184

2. Thomas $P$, Thomas M, Summer B, Dietrich K, Zauzig M, Steinhauser E et al (2011) Impaired woundhealing, local eczema, and chronic inflammation following titanium Osteosynthesis in a nickel and cobalt-allergic patient: a case report and review of the literature. https://insights.ovid.com/pubmed? pmid=21655880. Zugegriffen:5. Okt. 2017

3. DrugWatch Metallosis \& Metal Poisoning https://www.drugwatch.com/hip-replacement/ metallosis/.Zugegriffen:5.Okt. 2017

4. Nagels J, Stokdijk M, Rozing PM (2003) Stress shielding and bone resorption in shoulder arthroplasty. JShoulder Elbow Surg 12(1):35-39

5. Augat $P$, Simon U, Liedert A, Claes L (2005) Mechanics and mechano-biology of fracture healing in normal and osteoporotic bone. Osteoporos Int 16(2):S36-S43

6. Franchi M, Fini M, Giavaresi G, Ottani V (2005) Periimplant osteogenesis in health and osteoporosis. Micron 36(7-8):630-644

7. Soler-Botija C, Bagó JR, Llucià-Valldeperas A, Vallés-Lluch A, Castells-Sala C, Martínez-Ramos $C$ et al (2014) Engineered 3D bioimplants using elastomeric scaffold, self-assembling peptide hydrogel, and adipose tissue-derived progenitor cells for cardiac regeneration. Am J Transl Res 6(3):291-301

8. Takmakov P, Ruda K, Phillips KS, Isayeva IS, Krauthamer V, Welle CG (2015) Rapid evaluation of the durability of cortical neural implants using accelerated aging with reactive oxygen species. JNeural Eng 12(2):26003. https://doi.org/10.1088/ 1741-2560/12/2/026003

9. Linz C, Collmann H, Kübler A, Böhm H, Schweitzer T (2017) Patient-specific biodegradable implant in pediatric craniofacial surgery. J Craniomaxillofac Surg 45(8):1111-1111

10. Luef KP, Stelzer F, Wiesbrock F (2015) Poly(hydroxy alkanoate)s in medical applications. Chem Biochem Eng Q 29(2):287-297. https://doi.org/10. 15255/cabeq.2014.2261

11. Xiao W, Zaeem MA, Li G, Bal BS, Rahaman MN (2017) Tough and strong porous bioactive glassPLA composites for structural bone repair. J Mater Sci 52(15):9039-9054

12. Fu Q, Saiz E, Rahaman MN, Tomsia AP (2011) Bioactive glass scaffolds for bone tissue engineering: state of the art and future perspectives. Mater Sci Eng C 31(7):1245-1256. https://doi.org/10.1016/j. msec.2011.04.022

13. Martínez-Vázquez FJ, Miranda $P$, Guiberteau $F$, Pajares A (2013) Reinforcing bioceramic scaffolds with in situ synthesized $\varepsilon$-polycaprolactone coatings. J Biomed Mater Res A 101(12):3551-3559

14. Peroglio M, Gremillard L, Chevalier J, Chazeau L, Gauthier C, Hamaide T (2007) Toughening of bioceramics scaffolds by polymer coating. J Eur Ceram Soc 27(7):2679-2685

15. Baino F, Fiorilli S, Vitale-Brovarone C (2017) Composite Biomaterials based on sol-gel Mesoporous silicate glasses: a review. https://www-1ncbi1nlm-1nih-1gov-1pubmed.han.medunigraz.at/ pmc/articles/PMC5590434/. Zugegriffen: 6. Okt. 2017

16. León B, Jansen J (Hrsg) (2009) Thin calcium phosphate coatings for medical implants. http:// link.springer.com/10.1007/978-0-387-77718-4. Zugegriffen:6.Okt. 2017

17. Black J (2005) Biological performance of materials: fundamentals of biocompatibility, 4. Aufl. CRC Press, Boca Raton

18. Eliaz N, Metoki N (2017) Calcium phosphate Bioceramics: a review of their history, structure, properties, coating technologies and biomedical applications. Materials (Basel) 10(4):334. https:// doi.org/10.3390/ma10040334

19. Fagali NS, Grillo CA, Puntarulo S, Lorenzo de Mele MAF (2015) Cytotoxicity of corrosion products of degradable Fe-based stents: Relevance of $\mathrm{pH}$ and insoluble products. Colloids Surf B Biointerfaces 128:480-488. https://doi.org/10.1016/j.colsurfb. 2015.02.047

20. Moravej M, Mantovani D (2011) Biodegradable metals for cardiovascular stent application: interests and new opportunities. Int J Mol Sci 12(7):4250-4270. https://doi.org/10.3390/ ijms12074250

21. Hofstetter J, Martinelli E, Pogatscher S, Schmutz P, Povoden-Karadeniz E, Weinberg AM et al (2015) Influence of trace impurities on the in vitro and in vivo degradation of biodegradable $\mathrm{Mg}-5 \mathrm{Zn}-0.3 \mathrm{Ca}$ alloys. Acta Biomater 23:347-353

22. Purnama A, Hermawan $H$, Couet J, Mantovani D (2010) Assessing the biocompatibility of degradable metallic materials: State-of-the-art and focus on the potential of genetic regulation. Acta Biomater 6(5):1800-1807

23. Wu L, Feyerabend F, Schilling AF, Willumeit-Römer $R$, Luthringer BJC (2015) Effects of extracellular magnesium extract on the proliferation and differentiation of human osteoblasts and osteoclasts in coculture. Acta Biomater 27:294-304

24. Jähn K, Saito H, Taipaleenmäki H, Gasser A, Hort N, Feyerabend F et al (2016) Intramedullary Mg2Ag nails augment callus formation during fracture healing in mice. Acta Biomater 36:350-360

25. Zhang Y, Xu J, Ruan YC, Yu MK, O'Laughlin M, Wise $\mathrm{H}$ et al (2016) Implant-derived magnesium induces local neuronal production of CGRP to improve bone-fracture healing in rats. Nat Med 22(10):1160-1169. https://doi.org/10.1038/nm 4162

26. PichlerK, KrausT, MartinelliE, Sadoghi P, Musumeci G, Uggowitzer PJ et al (2014) Cellular reactions to biodegradable magnesium alloys on human growth plate chondrocytes and osteoblasts. Int Orthop 38(4):881

27. Myrissa A, Bräuer S, Martinelli E, Willumeit-Römer R, Gössler W, Weinberg A-M (2017) Gadolinium accumulation in organs of Sprague-Dawley ${ }^{\circledR}$ rats afterimplantation of abiodegradable magnesiumgadolinium alloy. Acta Biomater 48:521-529. https://doi.org/10.1016/j.actbio.2016.11.024

28. Myrissa A, Nezha AA, Lu Y, Martinelli E, Eichler J, Szakacs $G$ et al (2016) In vitro and in vivo comparison of binary Mgalloys and pure Mg. Mater Sci Eng C 61:865-874. https://doi.org/10.1016/j. msec.2015.12.064

29. Amerstorfer F, Fischerauer S, Fischer L, Eichler J, Draxler J, Zitek A et al (2016) Long-term in vivo degradation behavior and near-implant distribution of resorbed elements for magnesium alloys WZ21 and ZX50. Acta Biomater 42:440-450

30. Castellanie C, Lindtner RA, Hausbrandt P, Tschegg E, Stanzl-Tschegg SE, Zanoni G et al (2011) Boneimplant interface strength and osseointegration: Biodegradable magnesium alloy versus standard titanium control. Acta Biomater 7(1):432-440

31. Kraus T (2012) Magnesium alloys for temporary implants in osteosynthesis: In vivo studies of their degradation and interaction with bone. Acta Biomater 8(3):1230-1238

32. Fischerauer S (2013) In vivo degradation performance of micro-arc-oxidized magnesium implants: A micro-CT study in rats. Acta Biomater 9(2):5411-5420

33. Witte F, Hort N, Vogt C, Cohen S, Kainer KU, Willumeit R et al (2008) Degradable biomaterials based on magnesium corrosion. Curr Opin Solid State Mater Sci 12(5):63-72

34. Pogorielov M, Husak E, Solodivnik A, Zhdanov S (2017) Magnesium-based biodegradable alloys: Degradation, application, and alloying elements. Interv Med Appl Sci 9(1):27-38

35. Celarek A, Kraus T, Tschegg EK, Fischerauer SF, Stanzl-Tschegg S, Uggowitzer PJ et al (2012) PHB, crystalline and amorphous magnesium alloys: Promising candidates for bioresorbable osteosynthesis implants? Mater Sci Eng C 32(6):1503-1510

36. Grünewald TA, Rennhofer $\mathrm{H}$, Hesse B, Burghammer M, Stanzl-Tschegg SE, Cotte Metal (2016) Magnesium from bioresorbable implants: Distribution and impact on the nano-and mineral structure of bone. Biomaterials 76(Supplement C):250-260

37. Svedbom $A$, Hernlund $E$, Ivergård $M$, Compston J, Cooper C, Stenmark J et al (2013) Osteoporosis in the European Union: a compendium of country-specific reports. https://www-1ncbi1nlm-1nih-1gov-1 pubmed.han.medunigraz.at/ pmc/articles/PMC3880492/. Zugegriffen: 5. Okt. 2017

38. Kanis JA, McCloskey EV, Johansson H, Cooper C, Rizzoli R, Reginster J-Y et al (2013) European guidance for the diagnosis and management of osteoporosis in postmenopausal women. Osteoporos Int 24(1):23

39. Zhang H, Lewis $\mathrm{CG}$, Aronow MS, Gronowicz GA (2004) The effects of patient age on human osteoblasts' response to Ti-6Al-4V implants in vitro. J Orthop Res 22(1):30-38

40. Duarte PM, Neto JBC, Gonçalves PF, Sallum EA, Nociti júnior FH (2003) Estrogen deficiency affects bone healing around titanium implants: a histometric study in rats. Implant Dent 12(4):340-346. https://doi.org/10.1097/01. id.0000099750.26582.4b

41. Nicoli Aldini N, Fini M, Giavaresi G, Giardino R, Greggi T, Parisini $P$ (2002) Pedicular fixation in the osteoporotic spine: a pilot in vivo study on long-term ovariectomized sheep. J Orthop Res 20:1217-1224

42. Grünewald TA (2016) Reaction of bone nanostructure to a biodegrading Magnesium WZ21 implant - A scanning small-angle X-ray scattering time study. Acta Biomater 31:448-457

43. Pichler K, Fischerauer S, Ferlic P, Martinelli E, Brezinsek H-P, Uggowitzer PJ et al (2014) Immunological response to biodegradable magnesium implants. JOM (1989) 66:573-579 